\title{
Infección de prótesis total de rodilla y cadera. Epidemiología descriptiva, terapéutica y evolución en un hospital de segundo nivel durante 10 años
}

\author{
A. JOVER SÁENZ1', F. BARCENILLA GAITE, J. TORRES PUIG GROS ${ }^{3}$, \\ J. MAS ATANCE ${ }^{2}$, S. GARRIDO CALVO ${ }^{4}$, J. M. PORCEL PÉREZ ${ }^{1}$
}

Unidad Funcional para el control de la Infección Nosocomial. Servicios de 'Medicina Interna y ${ }^{2}$ Traumatología. Hospital Universitario Arnau de Vilanova. ${ }^{3}$ Sección de Epidemiología. Servicios Territoriales de Salud. Universitat de Lleida. ${ }^{4}$ ABS. Cruz RojaL'Eixample. Lleida

\begin{abstract}
TOTAL PROSTHETIC KNEE AND HIP JOINT INFECTION. DESCRIPTIVE EPIDEMIOLOGY, THERAPEUTICS AND EVOLUTION IN A SECONDARY HOSPITAL DURING TEN YEARS
\end{abstract}

\begin{abstract}
RESUMEN
Objetivo: Descripción epidemiológica retrospectiva en su clasificación, evolución y terapéuticas de las prótesis totales de cadera y rodilla infectadas entre las artroplastias realizadas desde el 1 de enero de 1994 al 31 de diciembre del 2003 en nuestro hospital.

Resultados: La tasa de infección global de artroplastias fue 2,4\% (intervalo de confianza del 95\% [IC 95\%], 1,6\% -3,2\%) sobre 40 casos de 1666 implantes articulares. La media de edad \pm desviación estándar (DE) fue de 69,5 $\pm 5,1$ años, en su mayoría mujeres (67,5\%). La localización más frecuente fue rodilla, 31 casos $(77,5 \%)$. En un $90,0 \%$ de los enfermos se filió la etiología, siendo un $64,0 \%$ cocos grampositivos (Staphylococcus plasmocoagulasa negativos y S. aureus), un $13,0 \%$ bacilos gramnegativos y flora mixta $(11,0 \%)$. Predominaron las infecciones agudas $(45,0 \%)$. El recambio articular en un tiempo tuvo una curación del $66,7 \%$ frente a un $83,3 \%$ en recambio en 2 tiempos.

Conclusiones: Los cocos gram positivos son los principales microrganismos en estas infecciones. Son necesarios estudios amplios multicéntricos para valorar la eficacia de las distintas técnicas quirúrgicas, los nuevos antibióticos y las terapias antibióticas combinadas.
\end{abstract}

\begin{abstract}
Objective: Epidemiological description, retrospective in its classification, evolution and therapeutics of total prosthesis knee and hip infected among the arthoplasties made between 1st of January 1994 and 31st of December 2003 in our hospital.

Results: The global infection rate was $2.4 \%$ (confidence interval of $95 \%$ [CI 95\%], 1.6\%-3.2\%) over 40 cases in 1,666 joint implants. The average age +/- standard deviation $(S D)$ was 69.5 years old $+/-5.1$ years, mainly in women $(67.5 \%)$. The most common location was the knee, 31 cases $(77.5 \%)$. In $90.0 \%$ of the patients the aetiology was identified: 64,0\% were gram-positive cocci (plasmacoagulase-negative staphylococci, and Staphylococcus aureus); $14.0 \%$ were gram-negative bacilli and mixed flora (11.0\%). Acute infections were found in most of the cases $(45,0 \%)$. The joint replacement had a healing rate of $66.7 \%$ in the first replacement and of $83.3 \%$ in the second replacement.

Conclusions: The gram-positive cocci are the main microorganisms in these $i$ nfections. More extensive studies about multiple aspects are necessary to evaluate the effectiveness of the different surgical techniques, the new antibiotics and the combined antibiotic therapies.
\end{abstract}

PALABRAS CLAVE: Infección. Prótesis articulares. Epidemiología.

KEY WORDS: Infection. Prosthetic joints. Epidemiology.

Jover Sáenz, A, Barcenilla Gaite F, Torres Puig Gros J, Mas Atance J, Garrido Calvo S, Porcel Pérez JM. Infección de prótesis total de rodilla y cadera. Epidemiología descriptiva, terapéutica y evolución en un hospital de segundo nivel durante 10 años. An Med Interna (Madrid) 2007; 24: 19-23.

\section{INTRODUCCIÓN}

En las últimas décadas, los reemplazamientos quirúrgicos articulares han supuesto una importante mejora en la capacidad funcional de los pacientes con artropatías. En nuestro país, se colocan anualmente alrededor de 30.000 prótesis articulares, la mayoría de rodilla y cadera, y en todo el mundo más de 1 millón. Sin embargo, no es una intervención exenta de complicaciones siendo de las más temidas la infección. En la actualidad, las tasas de infección rondan el 1,5\% en las pró- tesis de cadera y el $2,5 \%$ en las de rodilla (1). Su presencia, supone una importante disminución en la calidad de vida de los pacientes, un elevado coste económico ( $>50.000$ caso) $\mathrm{y}$ un sufrimiento humano que se traduce, en ocasiones, en una mortalidad de hasta un $18 \%$ (2). Para evitarlo, nuestra mejor arma es su prevención y sobre todo su conocimiento. Hasta el momento son escasos los estudios epidemiológicos prospectivos realizados al respecto, debido en parte a la baja incidencia de esta infección, lo que obliga a que éstos sean retrospectivos en la mayoría de ocasiones.

Trabajo aceptado: 11 de septiembre de 2006

Correspondencia: Alfredo Jover Sáenz. Unidad Funcional para el Control e la Infección Nosocomial. Hospital Universitario Arnau de Vilanova. Alcalde Rovira Roure, 80. Fax: 973248 754. 25198 Lleida. e-mail: ajover@arnau.scs.es 
Por otra parte, todavía no se ha establecido un tratamiento quirúrgico de elección ni antibiótico adecuado en tipo y duración, tras producirse la infección de la prótesis. La principal razón radica en la imposibilidad de realizar estudios comparativos de los diferentes procedimientos que permitan diseñar una correcta estandarización. En general, las alternativas quirúrgicas que se contemplan son: el desbridamiento y mantenimiento de la prótesis, la reimplantación de la artroplastia en 1 ó 2 tiempos, la artrodesis (rodilla) o la excisión completa (cadera) (3). En cuanto a la antibioterapia, parece necesario su combinación con la cirugía ya que como opción única se acompaña de un nivel de fracasos elevado.

Dado el interés que suscita la infección de prótesis articular, se pretende describir la experiencia a lo largo de 10 años en el tratamiento y evolución de estos enfermos en nuestro hospital.

\section{MÉTODOS}

\section{POBLACIÓN DEL ESTUDIO}

El Hospital Universitario Arnau de Vilanova de Lleida (España) es el centro asistencial de referencia de la provincia, dispone de 446 camas y ofrece una cobertura sanitaria a una población de 413.926 habitantes. Para el presente estudio, se investigaron retrospectivamente a todos los pacientes diagnosticados de infección de prótesis total de rodilla y cadera, implantada en el Servicio de Traumatología de este hospital, desde el 1 de enero de 1994 hasta el 31 de diciembre del 2003. Con los mismos, se planteó un estudio descriptivo basado principalmente en los aspectos de su tratamiento y evolución. Todos los pacientes, se obtuvieron del registro de artroplastias suministrado por la Unidad de Documentación.

\section{DISEÑO DEL ESTUDIO, DEFINICIONES, CRITERIOS DE INCLUSIÓN Y MÉTODOS DIAGNÓSTICOS}

Se definieron los casos como aquellos pacientes con artroplastia total de rodilla o cadera de nueva implantación (sólo el primer episodio) e infectada. La recogida de la información se realizó a partir de la historia clínica.

Para la vertiente descriptiva del estudio, se recogió información sobre los días de estancia, tipo y localización de la prótesis, métodos diagnósticos, clasificación de la infección, resultados microbiológicos, procedimientos quirúrgicos, tratamientos antibióticos y las complicaciones en el seguimiento. Los criterios diagnósticos de infección que se aceptaron fueron clínicos (fístula, presencia externa de signos inflamatorios o de pus en el acto operatorio) unidos al menos a uno de los siguientes: a) analíticos (elevaciones de la velocidad de sedimentación globular -VSG- y de la proteina $\mathrm{C}$ reactiva -PCR-); y b) imagen (radiolucencia de la interfase cementohueso, reabsorción en sacabocado o reacción laminada periostial en la radiografía simple, y/o hipercaptación en gammagrafías óseas con leucocitos marcados o con tecnecio 99-galio 67). Se utilizó la clasificación de Coventry (4) para definir la aparición de la infección, por ser la más utilizada en trabajos de nuestro entorno, dividiéndose en cuatro tipos: a) precoz, presencia de síntomas en los 3 primeros meses desde la cirugía; b) intermedia, cuando aparecieron entre los 4-12 meses; c) tardía a partir del 1 ${ }^{\text {er }}$ año; y d) hematógena (precoz o tardía), cuando el foco primario estuvo en otro origen no articular y los hemocultivos eran positivos.

En cuanto al diagnóstico microbiológico se requirió, en el caso del estafilococo plasmocoagulasa negativo, que estuviese presente en al menos 2 muestras, con el mismo antibiograma, recogidas intraquirófano. Para el resto de los microorganismos de probada patogenicidad, se consideró valorable con la identificación en una sola muestra. En el caso de los antibióticos, se recogieron las diferentes pautas parenterales y orales además de los días de utilización. En todos los pacientes infectados se revisaron sus visitas ambulatorias hasta un año después de finalizar el tratamiento. La evolución al año se clasificó según los siguientes términos: a) curación, desaparición de los síntomas y normalización de los reactantes de fase aguda, sin retirada del material de prótesis, tras un año de seguimiento; b) fracaso, persistencia durante ese año de los síntomas a pesar del tratamiento correcto o reaparición de los mismos tras completarlo incluyendo reactantes de fase elevados; y c) desconocida, imposibilidad de saber la evolución por la historia clínica.

\section{ANÁLISIS ESTADÍSTICO}

El análisis estadístico se realizó mediante recuentos y porcentajes para variables cualitativas y la media aritmética junto con la desviación estándar para las variables cuantitativas. La comparación de proporciones se realizó aplicando el test de la $\chi^{2}$ o la prueba exacta de Fisher, y el test de la t Student en la comparación de medias. La significación estadística se estableció a partir de un p $<0,05$. El análisis de los datos se efectuó con el paquete estadístico SPSS (versión 12.0).

\section{RESULTADOS}

En el periodo señalado se practicaron 1.666 artroplastias (930 rodillas y 736 caderas), 43 de la cuales se infectaron.

\section{TABLA I}

CARACTERÍSTICAS DEMOGRÁFICAS DE LOS PACIENTES Y TIPOS DE ARTROPLASTIAS

\begin{tabular}{lc}
\hline Características generales & $\begin{array}{c}\text { Casos }(\%) \\
(n=40)\end{array}$ \\
\hline
\end{tabular}

\begin{tabular}{|c|c|}
\hline Sexo & \\
\hline Hombre & $13(32,5)$ \\
\hline Mujer & $27(67,5)$ \\
\hline Edad media en años $( \pm D E)$ & $69,5 \pm 5,1$ \\
\hline Protesis & \\
\hline Rodilla & $31(77,5)$ \\
\hline Cadera & $9(22,5)$ \\
\hline Artroplastia & \\
\hline Rodilla & \\
\hline Link & $12(30,0)$ \\
\hline Press-Fit & $19(47,5)$ \\
\hline Cadera & \\
\hline AutoFit & $8(20,0)$ \\
\hline Mittelmeier & $1(2,5)$ \\
\hline
\end{tabular}

DE: Desviación estándar. 
Para el cómputo total sólo se contabilizaron 40 (31 rodillas y 9 caderas) al no poderse recuperar 3 historias clínicas. En todos los casos sólo existió un único episodio de infección. Las características demográficas de los pacientes y tipos de prótesis se muestran en la tabla I.

Para el conjunto del periodo de estudio, se obtuvieron unas tasas de infección global de artroplastias del 2,4\% (IC 95\%; $1,6 \%-3,2 \%$ ) y unas correspondientes a rodilla y cadera del $3,3 \%$ (IC $95 \% ; 2,1 \%-4,5 \%$ ) y $1,2 \%$ (IC 95\%; $0,4 \%-2,1 \%$ ), respectivamente. La estancia media postoperatoria fue de 17,3 $\pm 10,6$ días. Los principales microorganismos aislados fueron cocos grampositivos $(64,2 \%)$, cerca de la mitad de ellos Staphylococcus plasmocoagulasa negativos (Tabla II), sin que se observase una relación estadísticamente significativa entre los distintos gérmenes, lugar y tipo de prótesis.

En cuanto a los tipos de infección, 18 casos $(45,0 \%)$ fueron infecciones agudas, $12(30,0 \%)$ intermedias, $9(22,5 \%)$ crónicas y sólo una hematógena crónica $(2,5 \%)$ con unos tiempos medios desde la colocación de la prótesis hasta la infección de aproximadamente 1, 6, 19 y 24 meses, respectivamente. La infección aguda predominó en las prótesis de rodilla $(51,6 \%)$ mientras que la crónica lo hizo en las de cadera $(44,4 \%)$, aunque sin significación estadística. Por tipos de prótesis Press-fit en rodilla y Autofit en cadera fueron las más afectadas. El 66,7\% de las infecciones agudas (12 casos) fueron tratadas con desbridamiento y limpieza quirúrgica más antibioterapia prolongada, practicándose un explante de la prótesis en el resto de los pacientes y un reimplante en el $66,4 \%$ de éstos. El 75,0\% de los que se mantuvo la prótesis recidivaron. En las intermedias sólo en el 25,0\% (3 casos) se hizo un tratamiento conservador, en el resto se retiraron las prótesis recolocándose un $77,8 \%$, mientras que en las crónicas se extrajeron todas reimplantándose sólo el 50,0\%.

Algo más de la mitad de todos los recambios (9 sobre 16) se efectuaron en un primer tiempo y casi en su totalidad en la infección intermedia y crónica. Con este procedimiento, se

\section{TABLA II}

\begin{tabular}{lccc}
$\begin{array}{c}\text { RELACIÓN DE MICROORGANISMOS DETECTADOS EN LAS } \\
\text { PRÓTESIS DE RODILLA Y CADERA INFECTADAS }\end{array}$ \\
$\begin{array}{l}\text { PTR }(\%) \\
(n=31)\end{array}$ & $\begin{array}{c}\text { PTC }(\%) \\
(n=9)\end{array}$ & $\begin{array}{c}\text { Total } \\
(\%)\end{array}$ \\
\hline & & & \\
\hline $\begin{array}{l}\text { Microorganismos } \\
\text { Cocos grampositivos }\end{array}$ & & \\
Staphylococcus plasmocoagulasa & $15(37,5)$ & $1(7,7)$ & $16(30,2)$ \\
$\quad$ negativos & & & \\
Staphylococcus aureus & $5(12,5)$ & $3(23,1)$ & $8(15,1)$ \\
Streptococcus sp. & $5(12,5)$ & $3(23,1)$ & $8(15,1)$ \\
Enterococcus sp. & $2(5,0)$ & $0(0,0)$ & $2(3,8)$ \\
Bacilos gramnegativos & & & \\
Eschericha coli & $2(5,0)$ & $1(7,7)$ & $3(5,7)$ \\
Pseudomonas aeruginosa & $1(2,5)$ & $1(7,7)$ & $2(3,8)$ \\
Enterobacter cloacae & $1(2,5)$ & $0(0,0)$ & $1(1,9)$ \\
Pasteurella multocida & $0(0,0)$ & $1(7,7)$ & $1(1,9)$ \\
Otros* & $1(2,5)$ & $0(0,0)$ & $1(1,9)$ \\
Etiología polimicrobiana & $4(10)$ & $2(15,4)$ & $6(11,3)$ \\
Cultivo negativo & $4(10)$ & $1(7,7)$ & $5(9,4)$ \\
\hline
\end{tabular}

PTR: prótesis total de rodilla; PTC: prótesis total de cadera; $\mathrm{V}$ : varón; $M$ : mujer; *Mycobacterium tuberculosis. observó una curación completa en 6 pacientes $(66,7 \%)$ sobretodo cuando el agente causante de infección era Staphylococcus plasmocoagulasa negativo. Por el contrario, los recambios en un $2^{\circ}$ tiempo fueron mayoritarios en las infecciones agudas e intermedias (6 sobre 7) con una curación del 83,3\% (en 1 paciente no se supo la evolución), sin evidenciarse una evolución mejor respecto a ningún microorganismo.

De los 32 pacientes en los que se supo la evolución, en 17 casos $(53,1 \%)$ existió una curación completa. En el resto, 6 casos presentaron un fracaso durante el tratamiento y 9 una recidiva después del mismo, sometiéndose el 33,3\% del total de estos enfermos a una nueva colocación de prótesis y en un $50,0 \%$ a explante definitivo (Girdlestone). Los microorganismos que se documentaron en estos pacientes fueron Staphylococcus plasmocoagulasa negativos (6 casos), Streptococcus sp (4 casos), Staphylococcus aureus (3 casos), Pseudomonas aeruginosa ( 2 casos) y Enterococcus sp (1 caso).

En cuanto al tratamiento antibiótico, 37 pacientes $(92,5 \%)$ lo iniciaron en el hospital y $25(67,6 \%)$ lo prosiguieron posteriormente en el domicilio. La duración media del mismo fue de 31,4 días (rango; 5-77 días). La terapia antibiótica combinada estuvo presente en el 54,0\% de los tratamientos hospitalarios y un $28,0 \%$ de los domiciliarios, sin que se observase una evolución más favorable en los tratados de forma simple (43,7\% frente $42,9 \%)$. La relación de los antibióticos empleados se muestra en la figura 1. Ante la disparidad de tratamientos antibióticos no se pudo relacionar el grupo de fracasos con una determinada pauta antimicrobiana. Dos enfermos fallecieron antes de finalizar el tratamiento antibiótico a causa de un problema de índole no infecciosa.

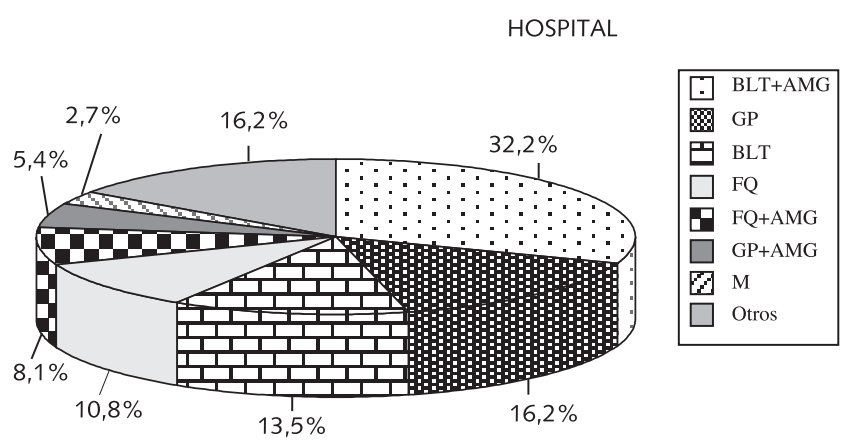

Duración media en días del antibiótico hospitalario: 16,5 días (rango; 0-62 días)

DOMICILIO

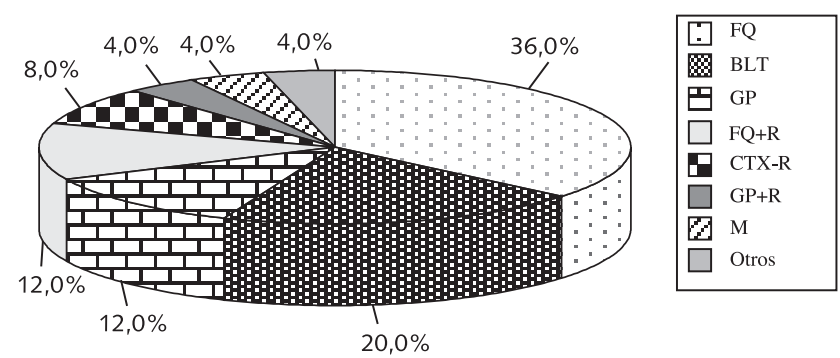

Duración media en días del antibiótico domiciliario: 14,8 días (rango; 0-40 días)

Fig. 1. Antimicrobianos utilizados en el tratamiento médico. 


\section{DISCUSIÓN}

Las tasas de infección tanto global como del lugar de prótesis que se han obtenido, son similares a las descritas en la literatura anglosajona (5) y alrededor de la mitad de las registradas en algunos trabajos de nuestro entorno (6). El espectro microbiológico encontrado es igual al de otras series (6-8) con un predominio de cocos grampositivos $(64,0 \%)$, en concreto Staphylococcus plasmocoagulasa negativos, seguido de bacilos gramnegativos $(13,0 \%)$ y flora mixta en un $11,0 \%$. Sin embargo no se han detectado anaerobios. Este hecho puede deberse al azar al ser una serie de escasa cuantía, sabiendo además que algunos de estos gérmenes como Propionibacterium acnes suele infectar más frecuentemente las artroplastias de hombro que las de otras localizaciones (9).

A lo largo de los últimos 30 años, la clasificación y pronóstico de la infección de prótesis articular se ha basado en criterios meramente temporales $(8,10)$. En este trabajo se ha utilizado la clasificación de Coventry, al ser bastante aceptada y comparable con otros estudios. No obstante, existe una falta de acuerdo en la literatura sobre cuál sería la clasificación más acertada, al considerar algunos autores (11) que los grupos de infección intermedia y tardía-crónica, por ser de etiología muy similar, podrían asociarse en un único grupo. Universalmente, en cualquiera de sus formas se contempla como tratamiento la combinación de una cirugía intervencionista junto a una terapia antimicrobiana prolongada. Como en nuestra serie, las estrategias que optan por la conservación de la prótesis en las infecciones crónicas, tras un desbridamiento y limpieza, tienen peores resultados a la hora de evitar recidivas oscilando sus tasas de curación entre el 8 y el $40 \%$, según los trabajos $(8,12)$. Por el contrario, la misma aptitud en la infección precoz eleva el nivel de curación hasta el $90-100 \%$ en pacientes seleccionados. Entre los criterios que deben cumplir estos pacientes destacan: tener una prótesis funcionante y estable, no existir una afectación de partes blandas y que el microorganismo causante no sea multirresistente o $S$. aureus (13). Posiblemente, el grupo de pacientes con infección aguda de este estudio no cumplían estos requisitos, lo que explicaría el elevado grado de recidiva observado $(75,0 \%)$. Por otra parte, el recambio de la prótesis en uno o dos tiempos se considera el tratamiento de elección en los pacientes que no cumplan criterios de una actuación conservadora. Clásicamente, el recambio en dos tiempos ha sido de elección, si bien esta tendencia no está claramente establecida en los últimos años. Langlais (14), en un artículo de revisión de distintas series publicado en 2003 cifraba en un estrecho margen la ventaja en las tasas de curación con el recambio en dos tiempos $(93,0 \%$ frente $86,0 \%)$. En ese mismo artículo, además se mencionaba un estudio retrospectivo europeo sobre 349 prótesis infectadas con un nivel de curación de un $88 \%$ en los recambios en un tiempo respecto a un $85 \%$ en dos tiempos. En nuestra serie, el porcentaje obtenido en los recambios en un tiempo es menor $(67,0 \%)$ posiblemente porque a los pacientes a los que se les practicó eran más complejos.

Por último, no está establecido que antibiótico o combinación de antibióticos son los más apropiados y durante cuanto tiempo tienen que administrarse $(15,16)$. Tampoco está definido el tiempo de antibioterapia parenteral antes de la secuenciación a vía oral, y el papel que tendrían los nuevos antiobióticos, con elevada biodisponibilidad oral, como las nuevas fluorquinolonas o el linezolid $(17,18)$. No obstante, en los últimos años se viene apuntando la necesidad de asociar rifampicina a otros antibióticos, en especial fluorquinolonas, en la infección provocadas por cocos grampositivos y su ventaja en la reducción del tiempo total de tratamiento (16). Zimmerli y cols. (13) demostraron, en un estudio aleatorizado y doble ciego, como en la infección estafilocócica aguda la asociación de ciprofloxacino con rifampicina administrada durante tres meses era superior a la monoterapia con un porcentaje de curación de un $100 \%$ frente a un $58 \%(\mathrm{p}<0,05)$. Sin embargo, el empleo de betalactámicos o cotrimoxazol, tanto en monoterapia como en combinación con rifampicina, no ha demostrado disminuir el número de recidivas, debido seguramente a la falta de penetración intracelular y a las menores concentraciones obtenidas por los primeros en los tejidos $(13,19,20)$. La variabilidad en las pautas antibióticas en el grupo de pacientes de este estudio y el escaso uso de la rifampicina con quinolonas $(12,0 \%)$ han podido influir en el bajo porcentaje de curación $(53,0 \%)$. No obstante, ésta y otras cifras se pueden ver minimizadas por las limitaciones propias de un estudio retrospectivo, al poder infravalorar otros potenciales factores de riesgo de infección y no disponer de la evolución completa de ciertos pacientes.

En resumen, el conocimiento de la infección de prótesis articular puede ayudar al clínico a establecer mecanismos de detección precoz y terapéuticas más adecuadas en su control evolutivo. Se necesitan estudios más amplios multicéntricos, prospectivos y aleatorizados, que evalúen cuáles serían los regímenes quirúrgicos y terapéuticos más eficaces en el tratamiento de las infecciones de prótesis articulares.

\section{Bibliografía}

1. Hanssen AD, Rand JA. Evaluation and treatment of infection at the site of a total hip or knee arthroplasty. J Bone Joint Surg 1998; 80: 910-22.

2. Ahlberg A, Carlsson AS, Lindgren L. Hematogenous infection in total joint replacement. Clin Orthop 1978; 137: 69-75.

3. Bernard L, Hoffmeyer P, Assal M, Vaudaux P, Schrenzel J, Lew D. Trends in the treatment of orthopaedic prosthetic infections. J Antimicrob Chemother 2004; 53: 127-9.
4. Coventry MB. Treatment in infections occurring in total hip surgery. Orthop Clin North Am 1975; 6: 991-1003.

5. Hanssen AD, Rand JA. Evaluation and treatment of infection at the site of a total hip or knee arthroplaty. J Bone Joint Surg 1998; 80-A: 910-22.

6. Gómez J, Rodríguez M, Baños V, Martínex L, Claver M, Ruiz J et al. Infección de prótesis articulares: epidemiología y clínica. Estudio prospectivo 1992-1999. Enferm Infecc Microbiol Clin 2002; 20: 74-7. 
7. Fitzgerald RH. Infected total hip arthroplasty: diagnosis and treatment. J Amer Acad Orthopaed Surg 1995; 3: 249-61.

8. Segawa H, Tsukayama DT, Kyle RF, Becker DA, Gustillo RB. Infection after total knee arthroplasty: a retrospective study of the treatment of eighty-one infections. J Bone Joint Surg Am 1999; 81: 1434-45.

9. Sperling JW, Kozak TK, Hanssen AD, Cofield RH. Infection after shoulder arthroplasty. Clin Orthop 2001; 382: 206-16.

10. Zimmerli W, Trampuz A, Ochsner PE. Prosthetic-joint infections: N England J Med 2004; 351: 1645-54.

11. Estrada R, Tsukayama D, Gustillo R. Management of total hip infections. A Prospective study of 108 cases. Orthop Trans 1993-94; 17: 1114-5.

12. Tattevin P, Cremieux AC, Pottier P, Huten D, Carbon C. Prosthetic joint infection: when can prosthesis salvage be considered?. Clin Infect Dis 1999; 29: 292-5.

13. Zimmerli W, Widmer AF, Blatter M, Frei R, Ochsner PE. Role of rifampin for treatment of orthopedic implant-related staphylococcal infections: a randomized controlled trial. Foreign-body infection (FBI) study group. Jana 1998; 279: 1537-41.

14. Langlais F. Can we improve the results of revision arthoplasty for infected total hip replacement?. J Bone Joint Surg Br 2003; 85: 637-40.
15. Bernard L, Hoffmeyer P, Assal M, Vaudax P, Screnzel J, Lew D. Trends in the treatment of orthopaedic prosthetic infections. J Antimicrob Chemother 2004; 53: 127-9.

16. Soriano A, García S, Ortega M, Almela M, Gallart X, Vila J et al. Tratamiento de la infección aguda de la artroplastia total o parcial de cadera con desbridamiento y régimen antibiótico oral. Med Clin (Barc) 2003; 121: 81-5.

17. Rimmele T, Boselli E, Breilh D, Djabarouti S, Bel JC, Guyot R, et al. Diffusion of levofloxacin into bone and synovial tissues. J Antimicrob Chemother 2004; 53: 533-5.

18. Bassetti M, Vitale F, Melica G, Righi E, Di Biagio A, Molfetta L, et al Linezolid in the treatment of gram-positive prosthetic joint infections. J Antimicrob Chemother 2005; 55: 387-90.

19. Brandt CM, Sistrucnk WW, Duffy MC, Hassen AD, Steckelberg JM, Llstrup DM, et al. Staphylococcus aureus prosthetic joint infection treated with desbridement and prosthesis retention. Clin Infect Dis 1997; 24: 914-9.

20. Drancourt M, Stein A, Argenson JN, Zannier A, Curvale G, Raoult D. Oral rifampin plus ofloxacin for tretament os staphylococcusinfected orthopedic implants. Antimicrob Agents Chemother 1993; 37: 1214-8. 